\title{
Mémoire et patrimoine: des récits et des affordances du patrimoine
}

\section{Memória e patrimônio: narrativas e affordances patrimoniais}

\section{Memory and cultural heritage: narratives and patrimonial affordances}

\author{
Joël Candau ${ }^{1}$ \\ Maria Leticia Mazzucchi Ferreira ${ }^{2}$
}

\begin{abstract}
RÉSUMÉ
Dans cet article, notre ambition est d'éclairer la notion d'éligibilité d'un objet patrimonial. Pour cela, nous proposons le concept d'affordance patrimoniale, l'éligibilité devenant d'autant plus probable que l'objet candidat à la patrimonialisation a cette qualité d'affordance. À partir de la distinction de deux types de savoirs et savoir-faire olfactifs observés dans divers milieux professionnels, nous tentons d'identifier une des conditions de la patrimonialisation de ce registre d'expertise dit "immatériel". Après avoir justifié notre typologie, nous montrons que le premier type de savoirs et savoir-faire olfactifs (type 1: cuisiniers, œnologues, parfumeurs, sommeliers) peut être facilement revendiqué comme un patrimoine parce que, de la part des professionnels, il offre une affordance narrative (il permet un récit de soi racontable et, de ce fait, recevable) alors que cela est impossible avec le second type d'expertise olfactive (type 2: employés de la morgue, fossoyeurs, infirmières, médecins légistes, sapeurs-pompiers, thanatopracteurs) qui n'offre pas une telle affordance.

Mots-clés: mémoire; usages du passé: patrimoine; savoir-faire; affordance.
\end{abstract}

DOI: $10.1590 / 0104-4060.43469$

1 Universidade Nice-Antipolis. Nice, França. 28 Avenue Valrose, 06103. E-mail: joel. candau@unice.fr

2 Universidade Federal de Pelotas. Instituto de Ciências Humanas. Pelotas, Rio Grande do Sul, Brasil. Rua Cel. Alberto Rosa, n 154, sala 121. CEP: 96020-220.E-mail: leticiamazzucchi@ gmail.com 


\title{
RESUMO
}

$\mathrm{Na}$ presente contribuição não buscamos responder à questão do conjunto de critérios contemporâneos que fundam a seleção ou a construção de um objeto patrimonial pois eles são múltiplos e variados. Nos propomos a evidenciar um critério de eligibilidade que, a nosso ver, não está documentado na literatura científica ou está apenas de maneira implícita. Propomos a noção de affordance patrimonial e defendemos a ideia supondo que tudo seja patrimonializável, da probabilidade que os diferentes candidatos que constituem esse tudo constituam esse processo dependendo da intensidade de sua affordance patrimonial. Parafraseando a célebre anedota, algo cínica, acerca de igualdade - todos os seres humanos são iguais mas alguns são mais do que os outros! - podemos dizer que todas as heranças do passado, seja próximo ou longínquo, são iguais face ao processo de patrimonialização, mas algumas são mais que outras em razão de seu grau de affordance.

Palavras-chave: memória; usos do passado; patrimônio; affordance.

\begin{abstract}
This article does not aim to address the modern criteria involved in the decision to declare a cultural object as official cultural patrimony, because those criteria are multiple and varied. This article proposes to highlight the criteria of eligibility, which is not documented clearly in our point of view, or is merely held to be implicit, in the existing academic discourse on cultural patrimony. This article proposes the concept of affordance of cultural heritage and defends the stance that, supposing that all cultural expressions can be potentially declared as cultural patrimony, the probability that a cultural expression be considered as such depends on its element of affordance. Paraphrasing the famous quote, however cynical, regarding equality - all men are created equal, but some are more equal than others! - we can state that all expressions of cultural heritage from the past, recent or distant, are equal in this process of cultural heritage, but some are more than others because of their degree of affordance.
\end{abstract}

Keywords: memory; uses of the past; cultural heritage; affordance.

Toutes les productions humaines, tous les savoir-faire humains, tous les héritages intangibles appelés improprement immatériels peuvent-ils devenir patrimoniaux? Cette question se pose au vu de la frénésie de patrimonialisation qui caractérise nombre de sociétés contemporaines, y compris quand il s'agit des pages les plus sombres de leur histoire. (SOSA; FERREIRA, 2014). Cette patrimonialisation est le produit d'un travail de mémoire qui, au fil du temps et selon des critères très variables, sélectionne certains éléments hérités du passé 
pour les ranger dans la catégorie des objets patrimoniaux. Cependant, ce travail de mémoire embrassant un passé de plus en plus large, ne peut-on supposer que tout - autrement dit, n'importe quoi - soit éligible pour devenir, un jour ou l'autre, du patrimoine?

La profusion actuelle d'éléments considérés comme patrimonialisables déborde complètement les principes des politiques patrimoniales nés au XIX siècle, fondés sur l'État-nation, puis sur des théories de la restauration et des pratiques visant à la reconnaissance et à la préservation du bien considéré. Tout au long du XX siècle, notamment jusqu'à la fin de la Seconde Guerre Mondiale, le patrimoine a suivi une trajectoire de valorisation des biens de pedra e cal, selon la conception de María Cecilia Londres da Fonseca (2005). L'introduction de nouveaux acteurs et objets dans le domaine patrimonial, observable à partir des années 1980, a ensuite élargi le concept de patrimoine en lui attribuant un nouveau statut et de nouvelles fonctions.

Au Brésil, cela se vérifie avec le développement de mouvements sociaux porteurs de revendications mémorielles, qui cherchent à attribuer une valeur patrimoniale aux rituels, traditions, récits, objets de la culture matérielle, lieux, paysages, etc. Penser le patrimoine dans la contemporanéité implique d'une part la prise en compte d'une pluralité de mémoires, de sujets, d'objets et de motivations, d'autre part le déplacement du regard de l'objet vers le processus de sa patrimonialisation et les enjeux sociaux qui lui sont associés.

Tentative de marquer le passage du temps en fabriquant des repères mémoriels, ce processus est une manière de concrétiser - de donner à voir - des expressions collectives de valorisation du passé et des croyances dans des mémoires partagées (métamémoires; CANDAU, 2011). Cette pratique de groupes ou de personnes organisés en mouvements de défense de leurs mémoires met en évidence ce que Daniel Fabre appelle des émotions patrimoniales (2013).

En ces temps mnémotropiques (CANDAU, 2011), de "culture de mémoire" (HUYSSEN, 2000), d'instauration d'un "régime patrimonial" (HAFSTEIN, 2007), où tout semble pouvoir se transformer en patrimoine, on peut alors se demander pourquoi certaines entités n'apparaissent jamais dans le casting du passé (APPADURAI, 2005, p. 67)? Dans la présente contribution, nous ne répondons pas à cette question dans sa globalité car il nous serait impossible d'aborder l'ensemble des critères contemporains qui fondent la sélection ou la construction d'un objet patrimonial. Ils sont en effet multiples et variés: liens affectifs, sentiment de l'urgence, souci d'édification des générations futures, sollicitations technologiques (pressions ou influences de la "cyberculture" et des dispositifs multimédia), intérêt religieux, intellectuel, esthétique, politique ou économique, ancienneté de l'objet ou bien encore la fameuse notion d'authenticité. Modestement, nous nous bornons à mettre en lumière un critère 
d'éligibilité qui, à notre connaissance, n'est pas documenté dans la littérature scientifique, ou alors seulement de manière implicite. Nous proposons la notion d'affordance patrimoniale et défendons l'idée que, à supposer que tout soit patrimonialisable, la probabilité que les différents candidats qui constituent ce tout entrent dans ce processus dépend de l'intensité de leur affordance patrimoniale. En paraphrasant la célèbre boutade, quelque peu cynique, sur l'égalité - tous les êtres humains sont égaux mais certains le sont plus que d'autres! -, nous pourrions dire que tous les héritages du passé, que celui-ci soit proche ou lointain, sont égaux face au processus de patrimonialisation, mais certains le sont davantage que d'autres, notamment du fait de leur degré d'affordance.

Après avoir brièvement précisé la notion d'affordance, nous donnons une illustration de ses effets patrimoniaux à partir de la distinction de deux types de savoirs et savoir-faire olfactifs observés dans divers milieux professionnels. Nous montrons que le premier type de savoirs et savoir-faire olfactifs (type 1: cuisiniers, œnologues, parfumeurs, sommeliers) peut être facilement revendiqué comme un patrimoine parce qu'il offre au discours des professionnels une prise narrative qui est une condition fondamentale de l'affordance patrimoniale. Ce type 1 permet en effet, pour ces professionnels, un récit de soi racontable notamment parce que le récit fait appel à un matrimoine olfactif - et, de ce fait, recevable. Cette prise narrative, qui donne lieu à un récit métamémoriel individuel puis collectif, est en revanche impossible avec le second type d'expertise olfactive (type 2: employés de la morgue, fossoyeurs, infirmières, médecins légistes, sapeurs-pompiers, thanatopracteurs). De ce fait, ce type 2 est un mauvais candidat à la patrimonialisation.

\section{L'affordance patrimoniale}

On doit la notion d'affordance au psychologue James Gibson (2014), lui-même s'étant inspiré de la notion d' "objets-signaux" ou "objets d'action" (des objets qui nous poussent à agir) ou de la notion de valence que l'on trouve chez un autre psychologue, Heinz Werner (1948). On peut décrire l'affordance comme la possibilité d'action qu'offre un objet par sa pure matérialité et qui varie en fonction de l'organisme qui interagit avec cet objet. Ainsi deux espèces animales perçoivent des "affordances" différentes dans le même objet. Une branche est affordante pour un oiseau, pas pour un cochon. De même, une chaise est affordante pour un être humain, en ce sens qu'elle a une valence qui incite à s'asseoir, mais elle n'est pas affordante pour un éléphant, sauf si c'est 
un animal de cirque ! Les caractéristiques saillantes de l'objet, c'est-à-dire ce qui en lui offre une prise perceptive, sont donc déterminées par les fonctions sensori-motrices de l'espèce et, au sein de celle-ci, des individus. L'affordance a probablement joué un grand rôle dès les premières productions culturelles humaines (NONAKA; BRIL; REIN, 2010) et elle est très présente dans les premiers stades du développement de l'enfant. (NIVELEAU, 2006). Compte tenu de l'éventail extraordinairement large des comportements chez Homo sapiens, l'affordance d'un objet n'est pas nécessairement univoque. Une bassine, par exemple, peut induire l'action de la remplir d'un liquide, mais elle peut aussi inciter un individu à s'en servir de tambour. Il y a donc une certaine plasticité dans l'affordance, que l'on peut s'attendre à retrouver avec les objets patrimoniaux. Dans une perspective quelque peu métaphorique, et dans un registre proche de ce que Kaufmann \& Clément (2007) appellent des "affordances sociales", nous soutenons que certains héritages du passé ont une affordance patrimoniale - ils induisent des actions de patrimonialisation, leur qualification patrimoniale va de soi - alors que d'autres héritages n'en ont pas ou très peu.

\section{Un patrimoine “immatériel” qui va de soi}

Par nature très intimes, les compétences sensorielles que sont la mémoire, les savoirs et les savoir-faire olfactifs en milieu professionnel représentent un défi pour l'anthropologue: peuvent-elles être partagées et transmises et, à ce titre, être qualifiées de sociales ou culturelles? Les résultats de nos recherches qui conjuguent méthodes ethnographiques et méthodes expérimentales (BACCINO et al., 2010; CANDAU, 2000, 2003, 2004, 2010; CANDAU; JEANJEAN, 2006; CANDAU; WATHELET, 2006, 2011) attestent ce partage et cette transmission: il existe des cultures olfactives au sein de professions reconnues pour leur expertise dans ce domaine (type 1) mais aussi parmi des professions (type 2) moins réputées que d'autres pour leurs aptitudes dans ce registre sensoriel.

Parmi les professions que nous venons de citer, on peut distinguer des savoirs et savoir-faire olfactifs dont l'appartenance à un patrimoine dit "immatériel" ${ }^{\prime 3}$ ne fait pas vraiment débat (type 1) et d'autres pour lesquels la réponse

3 Nous mettons le mot entre guillemets car il s'agit d'une notion controversée - pour de multiples raisons: voir par exemple Bortolotto 2011 - notamment du fait de sa connotation dualiste. Ce patrimoine, comme l'a justement noté Michel Van Praët (2002), relève "bien de différents états de la matière". 
est problématique (type 2$)^{4}$. Dans la première catégorie, celle où la dimension patrimoniale des savoirs et savoir-faire olfactifs semble s'imposer naturellement, on doit évidemment ranger toute la filière de la parfumerie. Il existe des musées de la parfumerie (Musée International de la Parfumerie à Grasse, Osmothèque à Versailles, etc.), des écoles (Institut Supérieur International du Parfum, de la Cosmétique et de l'Aromatique alimentaire à Versailles; Grasse Institute of Parfumery; École supérieure du Parfum à Paris), des expositions, d'innombrables publications dont certaines traitent explicitement du lien entre olfaction et patrimoine (BOILLOT et al., 2004), bref tout un dispositif institutionnel mettant en valeur ce qui est considéré comme un patrimoine et que, dans cet esprit, on appelle "l'art des parfums". En avril 2015, la ville de Grasse a d'ailleurs déposé un dossier auprès de l'UNESCO - intitulé Les savoir-faire lies au parfum en pays de grasse: la culture de la plante a parfum, la connaissance des matières premières naturelles et leur transformation, l'art de composer le parfum - en vue de l'inscription de ces savoir-faire au patrimoine culturel immatériel de l'humanité. Il nous semble aussi que la proposition de ranger dans cette même catégorie les savoirs et savoir-faire olfactifs relatifs au vin (sa fabrication et sa consommation) devrait être peu contestée. Après tout, il existe une Chaire UNESCO intitulée Culture et Traditions $d u$ Vin, dénomination qui renvoie bien à une dimension patrimoniale, en l'occurrence un patrimoine national. Sans que cela constitue l'essentiel de l'activité de cette chaire, la question de l'expertise olfactive y est parfois évoquée. Il en va de même avec les savoirs et savoir-faire olfactifs relatifs à la cuisine. Comme on le sait, Le repas gastronomique des Français a été inscrit par l'UNESCO sur la liste représentative du patrimoine culturel immatériel de l'humanité en 2010, année d'ailleurs où furent également inscrits L'art du pain d'épices en Croatie du Nord, La cuisine traditionnelle mexicaine - culture communautaire, vivante et ancestrale, le paradigme de Michoacán et La diète méditerranéenne. Voici un extrait de la description du repas gastronomique des Français sur le site de l'UNESCO:

Le repas gastronomique met l'accent sur le fait d'être bien ensemble, le plaisir du goût, l'harmonie entre l'être humain et les productions de la nature. Parmi ses composantes importantes figurent: le choix attentif des mets parmi un corpus de recettes qui ne cesse de s'enrichir; 1'achat de bons produits, de préférence locaux, dont les saveurs s'accordent

4 Ici, nous examinons uniquement la possibilité de l'existence d'un patrimoine olfactif au sein des professions citées. La question de l'existence d'un tel patrimoine dans d'autres contextes (par exemple, dans le milieu familial, avec transmission entre générations) est autrement plus difficile. Sur ce point, voir Schaal (2004); Ferendzi et al. (2008, 2010); Wathelet (2009, 2012). 
bien ensemble; le mariage entre mets et vins; la décoration de la table; et une gestuelle spécifique pendant la dégustation (humer et goûter ce qui est servi à table). (UNESCO, <http://www.unesco.org/culture/ich/ fr/RL/00437>.).

On le voit, l'olfaction est explicitement évoquée dans cette description, pas seulement par le verbe "humer" mais aussi par les mots "saveurs", "goûter" et "goût" puisque le sens gustatif est en réalité à $90 \%$ un sens olfactif dont la spécificité est de passer par la voie rétronasale et non par la voie orthonasale.

Enfin, on peut noter que les trois catégories de savoir et savoir-faire olfactifs que nous venons d'évoquer (relatives aux parfums, au vin et à la gastronomie) relèvent indubitablement de l'un voire de deux des cinq domaines du patrimoine culturel "immatériel" définis dans l'article 2.2 de la Convention pour la sauvegarde de ce patrimoine acceptée le 17 octobre 2003 par la Conférence générale de l'UNESCO: les savoir-faire liés à l'artisanat traditionnel; les pratiques sociales, rituels et événements festifs.

Ces savoirs et savoir-faire olfactifs sont donc d'ores et déjà considérés comme un patrimoine. Dès lors, la question de leur qualification patrimoniale ne se pose pas si on admet une définition anthropologique de ce patrimoine, i.e. une définition privilégiant le point de vue emic (celui des membres de la société étudiée et des institutions dont ces individus se sont dotés), opposé au point de vue etic (qui est l'interprétation théorique du chercheur): est patrimoine ce qui est considéré comme tel par une population donnée. Nous savons bien que pour d'autres disciplines cette définition assez proche de l'esprit de la convention de l'UNESCO (faible théoriquement, mais pragmatique et de ce fait très commode!) est loin d'être satisfaisante. Elle n'est ni ontologique ni analytique, mais elle correspond au parti pris de l'anthropologue qui consiste à prendre au sérieux les catégories que construisent "les gens", y compris quand il y a non seulement hétérogénéité rédhibitoire des membres de ces catégories mais aussi, éventuellement, une ontologie défaillante lorsque la catégorie renvoie soit à des choses qui ne sont pas ce qu'il est dit qu'elles sont, soit à des choses inexistantes. Bref, l'anthropologue n'est ni gêné ni effrayé par les interprétations "sauvages", "naïves" ou "profanes" du patrimoine. Nous risquons cet argument parce que si, par exemple, nous cherchions à appliquer aux savoirs et savoir-faire olfactifs relatifs aux parfums, au vin et à la gastronomie, les critères de "mise en patrimoine" proposés par Nathalie Heinich: valeur d'authenticité (ontologique ou catégorielle et historique), valeur de présence ${ }^{5}$, valeur de beauté et valeur de

5 “'...] qui s'attache à la proximité avec une personne, au sentiment d'une rencontre, d'un 
rareté (HEINICH, 2012, p. 26), on s'apercevrait sans doute de l'impossibilité de la tâche. Certes, il est fort possible que les critères de Heinich (ou d'autres) soient peu satisfaisants ou faiblement opératoires, mais il est possible aussi, et nous sommes enclins à le penser, qu'ils soient révélateurs du flou de cette catégorie du patrimoine lorsqu'elle est appliquée aux trois domaines (parfums, vin, gastronomie) que nous examinons ici. Une fois encore, peu importe pour l'anthropologue: ils sont considérés comme constituant un patrimoine et on se doit d'écarter toute approche normative consistant à dire qu'ils n'en sont pas un!

Dans ce qui suit, nous n'allons donc pas discuter de la qualification patrimoniale de ces savoirs et savoir-faire olfactifs (de type 1) mais simplement évoquer une de leurs caractéristiques qui renvoie à la notion de "matrimoine olfactif". Après cela, nous nous engagerons dans une tâche plus complexe: déterminer pourquoi l'appartenance au patrimoine "immatériel" des savoirs et savoir-faire olfactifs de type 2 ne présente pas le même caractère d'évidence que ceux de type 1. Au-delà de ce constat, la question que nous posons est de savoir s'il y a une différence de nature entre les savoirs et savoir-faire de type 1 et de type 2 . Nous faisons l'hypothèse que cette différence existe, notamment en termes d'affordance, et qu'elle joue un rôle décisif dans le processus de patrimonialisation. Pour une raison que nous allons expliquer, les professionnels de type 1 ont réussi à institutionnaliser un discours patrimonialisant, ce qui n'est pas du tout le cas dans les professions de type 2 .

\section{Les savoirs et savoir-faire olfactifs de type 1: patrimoines/matrimoines olfactifs}

Quand on considère la mémoire, les savoirs et les savoir-faire olfactifs dans le domaine des parfums, du vin et de la gastronomie, on s'aperçoit que ce patrimoine "immatériel" repose largement sur des matrimoines olfactifs, pour reprendre une expression de Benoist Schaal (2004). Du point de vue de l'ontogenèse, plusieurs recherches en biologie mais aussi en sciences et neurosciences cognitives et en anthropologie suggèrent qu'un contenu chimiosensoriel est transmis par la mère à l'enfant in utero puis durant toute la petite enfance. Ce que Schaal (2004) appelle "l'écologie osmique" d'un individu est largement constituée d'apprentissages olfactifs faits pendant l'enfance dont certains sont conservés jusqu'à l'âge adulte. L'un d'entre nous a obtenu des résultats allant

contact avec les êtres liés à cet objet”. (HEINICH, 2012, p. 26). 
dans ce sens lors d'une enquête par questionnaire auprès de 503 étudiants de l'Université de Nice Sophia Antipolis, entre 1996 et 1999. Dans leurs réponses, on compte essentiellement (à raison de $85 \%$ ) des souvenirs olfactifs à la tonalité agréable, renvoyant à l'enfance, aux vacances, aux voyages, à la nature (mer, montagne, campagne) ${ }^{6}$ et à la famille (odeurs et parfums des parents et grands-parents, maison familiale, plats cuisinés le dimanche, pâtisserie). Si l'on en juge d'après cette forte présence de l'enfance, les souvenirs olfactifs de cette période de la vie, notamment entre 6 et 10 ans (CHU; DOWNES, 2000), pourraient constituer des sortes d'empreintes conservées tout au long de notre existence. Des références à de telles empreintes olfactives apparaissent à plusieurs reprises dans le discours des professionnels du parfum, du vin et de la gastronomie.

Francis Kurkdjian, parfumeur-créateur, se souvient avec précision des odeurs d'huile, de machine à coudre, de craie de tailleur mais aussi des odeurs alimentaires qui toutes habitaient le petit appartement de ses grands-parents à Vincennes. (LE MINISTÈRE DE LA CULTURE ET DE LA COMMUNICATION, 2012, p. 18). Pour expliquer sa vocation de "nez", Mathilde Laurent évoque des chocs olfactifs de son enfance dans le maquis corse. Elle ajoute que "ce n'est pas l'odeur qui est la source d'inspiration, mais l'histoire qu'elle crée autour”. (LE MINISTÈRE DE LA CULTURE ET DE LA COMMUNICATION, 2012, p. 19). Françoise Caron, parfumeur également, est issue d'une famille où s'exerçait ce métier. Elle explique qu'elle a "toujours vécu au milieu des fleurs, des odeurs et des parfums". (LE MINISTÈRE DE LA CULTURE ET DE LA COMMUNICATION, 2012, p. 19).

Dans nos propres enquêtes (CANDAU, 2000), nous avons recueilli de très nombreuses données similaires. Chez les parfumeurs, l'image associée au stimulus olfactif renvoie souvent à la biographie personnelle, telle substance évoquant l'odeur de la cuisine de la grand-mère, telle autre une odeur de bouquins qu'on ouvre ou encore la quincaillerie que notre informateur avait l'habitude de visiter enfant. Le fait d'avoir vécu au contact de sollicitations olfactives propres à une région laisse entrevoir la possibilité d'une focalisation culturelle de la sensibilité olfactive dès le stade de la mémorisation: je reconnais plus facilement l'odeur du thym et du cyprès, constate un parfumeur, parce que j'ai évolué dans cet environnement olfactif dans mon enfance. C'est le vécu qui ressort, déclare un œnologue et qui, toujours, fait revenir aux mêmes critères analytiques et sur la même orientation de vocabulaire. Un autre œnologue associe systématiquement l'odeur de pêche au verger de son grand-père qu'il parcourait enfant. Un autre assimile l'odeur du musc à celle du vieux linge de

6 Ceci est congruent avec les observations faites de son côté par Anne Muxel (1996, p. 105).

7 Les propos de nos informateurs sont toujours mentionnés en italiques. 
maison qu'il découvrait tout enfant dans une malle ${ }^{8}$ entreposée dans le grenier de sa grand-mère. Les souvenirs d'enfance, en particulier les recettes maternelles, sont également omniprésents dans la mémoire culinaire des cuisiniers. Un des chefs avoue: toute ma cuisine est basée là-dessus. Un autre explique son refus d'intégrer certains plats sur sa carte par des dégoûts alimentaires remontant à son enfance. Comme chez les parfumeurs, les œnologues et les cuisiniers, la biographie personnelle joue un grand rôle dans la mémoire professionnelle du sommelier. C'est le cas, par exemple, quand l'un d'entre eux rattache des parfums de fruits rouges aux cerises Burlat de sa grand-mère ou à la tarte aux framboises de sa mère.

Retenons de ces quelques exemples que les savoirs et savoir-faire olfactifs de type 1 sont presque systématiquement inscrits par les professionnels dans un récit autobiographique - ce que certains de nos informateurs appellent un "vécu" - fortement pénétré de souvenirs d'enfance et, notamment, de souvenirs en rapport avec la maison familiale, le parfum de la mère, la cuisine de la grand-mère, le linge de maison, bref un univers non exclusivement mais largement féminin, ce qui semble justifier l'évocation de matrimoines olfactifs. C'est, pour une grande part, cette possibilité d'ancrage dans un récit autobiographique et, particulièrement, dans la représentation de matrimoines olfactifs, qui va donner consistance au sentiment d'une identité professionnelle (celle de parfumeur-créateur, de Chef, d'œnologue, de sommelier) et qui va permettre de revendiquer cette identité comme relevant d'un patrimoine. Les savoir-faire olfactifs propres aux autres professions qui ont fait l'objet de nos enquêtes offrent-ils la même prise narrative?

\section{Les savoirs et savoir-faire olfactifs de type 2: un discours patrimonialisant impossible}

Avant de répondre à cette question, nous devons évoquer rapidement trois aspects anthropologiquement pertinents du travail de mémoire: protomémoriels, mémoriels et métamémoriels. Nous ne développerons pas ici les deux premiers aspects dont nous donnons simplement une définition succincte: la protomémoire est le fruit d'un apprentissage "par corps", pour paraphraser Bourdieu (1997, p.

8 Sur le fort pouvoir évocateur des odeurs de vieux vêtements retrouvés dans une malle ou une armoire, voir Boris Cyrulnik (1993, p. 24), Gibbons (1986); Gilbert \& Wysocki (1987). 
163), qui le plus souvent agit le sujet à son insu; la mémoire, dite de haut niveau, est la faculté cognitive fondée sur le rappel et la reconnaissance.

La notion de métamémoire mérite qu'on s'y attarde davantage car cette ressource cognitive structure fortement les propos de la plupart de nos informateurs de type 1, comme nous l'avons montré supra, et elle est peu consistante voire inexistante chez nos informateurs de type 2. La métamémoire est d'une part la représentation qu'un individu se fait de sa propre mémoire, la connaissance qu'il en a, d'autre part ce qu'il en dit. Le niveau métamémoriel est celui d'un regard réflexif sur les processus mémoriels qu'un individu est capable de mobiliser dans l'accomplissement d'une tâche, ou qu'il se croit capable de mobiliser. Lorsqu'on passe de l'individu au groupe, la métamémoire est une dimension essentielle du sentiment d'une intersubjectivité mémorielle. C'est parce que nous avons conscience de ce que nous partageons, et parce que nous en parlons, que nous sommes capables de revendiquer une mémoire commune. Toutefois, il n'est pas nécessaire que la conscience du partage renvoie à un partage réel pour que naisse cette revendication. En effet, si la revendication d'une mémoire partagée se fonde toujours sur le postulat du partage, ce partage peut être réel ou imaginaire. C'est la raison pour laquelle le sentiment d'une mémoire partagée - tout comme le sentiment de partager un patrimoine immatériel ${ }^{9}$ - est parfois illusoire.

Cette dimension métamémorielle fait défaut au discours des professionnels de type 2. En premier lieu, ce discours n'est pas nourri ou relayé par des dispositifs institutionnels ad hoc (locaux, nationaux ou internationaux) comme il en existe dans le domaine de la parfumerie, du vin ou de la gastronomie et dont la mission est de dire (dimension performative) que ce type d'expertise olfactive relève d'un patrimoine. On peut alors se demander pourquoi ces savoirs et savoir-faire olfactifs ne peuvent prendre appui sur des dispositifs institutionnels. L'explication que nous proposons est qu'en l'absence de narrations possibles, de références ostentatoires à un "vécu" professionnel, de récits relatifs à ces savoirs et savoir-faire olfactifs, i.e. en l'absence d'un discours métamémoriel collectif, la création de tels dispositifs est impossible. Dans les professions de type 2 , ce discours est généralement peu consistant ou inaudible. Son indigence peut elle-même s'expliquer par l'absence de prises narratives, c'est-à-dire par l'impossibilité d'ancrer la narration dans un récit de soi (un niveau métamémoriel davantage personnel, individuel) et notamment dans un récit de soi où pourrait trouver sa place un matrimoine olfactif. Contrairement aux parfumeurs, cuisiniers, œnologues ou sommeliers, les souvenirs purement biographiques extérieurs au métier sont absents de l'expertise olfactive des thanatopracteurs,

9 Pour Chiara Bortolotto (2011, p. 24), "le patrimoine immatériel incarne la forme la plus extrême d'objectivation métaculturelle de la culture entendue dans son acception anthropologique". 
médecins légistes, fossoyeurs, infirmières ${ }^{10}$. Cela est facilement compréhensible. On peut très volontiers expliquer et raconter comment on est devenu un "nez", un grand chef de cuisine, un sommelier ou un œnologue en faisant référence à une sorte de roman familial (un héritage de dons supposés ou d'un habitus domestique particulier) et personnel (des expériences sensorielles relevant d'une mémoire autobiographique sur le modèle du syndrome de Proust). C'est ce que font beaucoup de professionnels dans ce domaine. En revanche, cela est quasiment impossible pour un thanatopracteur, une infirmière, un employé de la morgue ou un fossoyeur, en particulier parce que les odeurs de la mort, de la charogne et de la maladie peuvent difficilement être intégrées - et valorisées - dans un tel roman. Pour s'en convaincre, il suffit de comparer les quelques descriptions de stimuli olfactifs recueillies auprès de professionnels de type 1 que nous avons données plus haut et qui font songer à une sorte de jardin d'Eden (il y est question de fleurs, de thym, d'odeurs de verger, de cuisine, de linge de maison, de grenier, etc., soit des stimuli olfactifs généralement qualifiés de plaisants) avec celles que nos informateurs de type 2 ont faites des odeurs de la mort. Ils évoquent par exemple des corps qui se liquéfient, coulent, suintent, exsudent, refoulent et sont assimilés, quand ils sont bien avancés, à du jus, de la sauce, du bouillon. Nous pourrions multiplier les exemples de ce type. Ainsi, nous avons recueilli des descriptions extrêmement riches des odeurs d'enfants morts (enfants mort-nés, morts subites, accidents), qui renvoient à des compétences olfactives probablement aussi subtiles que celles qui fondent l'expertise d'un parfumeur ou d'un œnologue et qui, indéniablement, sont constitutives d'une culture olfactive. Cependant, cette culture ne peut accéder au statut patrimonial car, comme on l'imagine sans peine, non seulement ce ne sont pas des choses dont on peut parler facilement en public, contrairement à la description d'un Pomerol, du parfum Eau sauvage ou de l'odeur d'un civet de lièvre, mais il est extrêmement difficile pour un individu d'intégrer ces compétences olfactives - l'identification d'une putative odeur spécifique des enfants morts - dans un récit de soi (notamment parce que dans ce cas, sans la prise narrative qui conditionne l'affordance patrimoniale, la pénibilité cognitive de la narration est énorme), alors qu'il est si facile de dire que l'on est sensible aux arômes des vins, aux senteurs d'un parfum ou à la complexité de l'odeur d'un civet parce qu'on a vécu enfant dans un environnement peuplé d'odeurs,

10 C'est sans doute moins vrai pour les sapeurs-pompiers qui ne sont pas systématiquement exposés aux odeurs auxquelles les professions que nous venons de citer sont quotidiennement confrontées; dans leur métier, ils exercent leur odorat sur une palette d'odeurs beaucoup plus large, non limitée au versant négatif de l'espace hédonique. 
parce qu'on a été éduqué à cela, parce qu'on a (acquisition ou héritage) le goût des belles et bonnes choses, etc.

\section{Remarques conclusives}

On ne croit pas seulement ce qu'on croit, on pense et on dit aussi qu'on le croit, ce qui donne davantage d'autorité à ce qui est cru, autorité qui pourra ensuite se manifester sous des formes institutionnelles. Encore faut-il pouvoir le penser et le dire. On aura d'autant plus de facilité à le faire que ce qui est cru pourra être intégré dans un récit, et plus précisément dans un récit de soi riche d'expériences sensorielles supposées constituer un habitus familial et personnel et qui, à ce titre, pourront être valorisées.

Le patrimoine, comme tout partage mémoriel supposé, est avant tout un discours sur le partage qui travaille sans relâche à entretenir la croyance dans sa réalité. La notion même de patrimoine est d'ailleurs très largement, pour les acteurs du patrimoine dont nous faisons partie, une ressource rhétorique visant à renforcer la croyance dans son existence. Souvent, nous confondons le fait de dire, d'écrire ou de penser que nous avons un patrimoine avec l'idée que ce qui est dit, écrit ou pensé rend compte d'un partage réel. Bref, nous confondons le fait du discours avec ce qu'il est supposé décrire. Cette confusion a une fonction sociale importante: elle renforce dans les consciences individuelles le sentiment du partage, de l'identité et de la continuité. Nous avons défendu ici l'idée que, pour le patrimoine culturel "immaterial" plus particulièrement, ce discours n'est possible que s'il peut, plus ou moins, s'ancrer dans un récit de soi, condition de l'affordance patrimoniale. C'est le cas du discours tenu sur les savoirs et savoir-faire olfactifs de type 1 (parfumerie, vin, gastronomie), avec des références explicites à des matrimoines olfactifs intégrés dans un roman familial. Ce n'est pas le cas des savoirs et savoir-faire olfactifs dans les autres professions que nous avons évoquées (type 2). Parce qu'ils n'offrent pas de prise narrative, ces savoirs et savoir-faire ne présentent pas d'affordance patrimoniale, ils n'induisent pas une action de patrimonialisation. Il est possible que dans les deux cas la croyance dans le partage d'un patrimoine soit illusoire, mais dans le premier cas cette croyance peut être aisément partagée - qui, parmi nous, ne se reconnaîtra pas dans un récit évoquant les odeurs d'un grenier où l'on jouait enfant ou celles de la daube préparée par la grand-mère? - alors que dans le second cas rien de semblable n'advient. 
Concluons par une remarque de portée générale. Pour qu'il y ait patrimoine, il faut que les caractéristiques de l'entité (matérielle ou "immatérielle") candidate à la patrimonialisation puissent être considérées par les membres d'un groupe comme la trace d'une histoire racontable, comme le support d'un récit de soi qui, idéalement, sera reçu, entendu, approprié et partagé par les destinataires de cette histoire et ainsi inséré dans une histoire collective (familiale ou communautaire). De ce point de vue, toutes les entités candidates à la patrimonialisation ne sont pas égales. Dans le domaine du patrimoine dit "immatériel” bien plus que dans le domaine du patrimoine matériel, la réussite du processus dépend tout autant sinon plus de la possibilité d'une appropriation individuelle des récits que des dynamiques d'appropriation collective (logiques communautaires, politique des institutions, groupes d'acteurs, associations locales, intérêts économiques, etc.). Pour cette raison, il nous semble que le patrimoine matériel tient pour une grande part sa qualité patrimoniale à son affordance physique - en ce sens qu'il induit une représentation patrimoniale par sa seule matérialité, par sa simple visibilité - alors que le patrimoine "immatériel" tient cette même qualité de son affordance narrative: seules les entités qui donnent prise à un récit puissant, individuel puis collectif, ont des chances d'acquérir et de conserver une consistance patrimoniale.

\section{RÉFÉRENCES}

APPADURAI, A. Les conséquences culturelles de la globalisation. Paris: Payot \& Rivages, 2005.

BACCINO, Thierry; CABROL-BASS, Daniel; CANDAU, Joël; MEYER CANDICE, Scheer Tobias; VUILLAUME, Marcel; WATHELET, Olivier. Sharing an Olfactory Experience: The Impact of Oral Communication. Food Quality and Preference, v. 21, n. 5, p. 443-452, 2010.

BORTOLOTTO, Chiara. Le trouble du patrimoine culturel immatériel. Terrain [En ligne]. Le patrimoine culturel immatériel, 2011. <http://terrain.revues.org/14447>.

BOILLOT, Francine; GRASSE, Marie-Christine; HOLLEY, André (Éd.). Olfaction \& patrimoine: quelle transmission? Aix-en-Provence: Édisud, 2004.

BOURDIEU, Pierre. Méditations pascaliennes. Paris: Seuil, 1997.

CANDAU, Joël. Mémoire et expériences olfactives. Anthropologie d'un savoir-faire sensoriel. Paris: PUF, 2000. 
CANDAU, Joël. El lenguaje natural de los olores y la hipótesis Sapir-Whorf. Revista de antropología social, v. 12, p. 243-259, 2003.

CANDAU, Joël. The Olfactory Experience: constants and cultural variables. Water Science \& Technology, v. 49, n. 9, p. 11-17, 2004.

CANDAU, Joël. Shared memory, odours and sociotransmitters or: "Save the interaction!’. Outlines. Critical Practice Studies, v. 12, n. 2, p. 29-42, 2010.

CANDAU, Joël. Memória e Identidade. Traduction Maria Leticia Ferreira. São Paulo: Editora Contexto, 2011.

CANDAU, Joël; JEANJEAN, Agnès. Des odeurs à ne pas regarder. Terrain, v. 47, p. 51-68, 2006.

CANDAU, Joël; WATHELET, Olivier. Le nez cardinal. Sigila, v. 18, p. 49-61, 2006.

CANDAU, Joël; WATHELET, Olivier. Les catégories d'odeurs en sont-elles vraiment? Langages, v. 181, p. 37-52, 2011.

CHU, Simon; DOWNES, John Joseph. Long live Proust: the odour-cued autobiographical memory bump. Cognition, v. 75, p. B41-B50, 2000.

CYRULNIK, Boris. Les nourritures affectives. Paris: Odile Jacob, 1993.

FABRE, Daniel (Éd.). Émotions patrimoniales. Paris: Éditions de la Maison des Sciences de l'homme, 2013.

FERDENZI, C.; WATHELET, O.; SCHAAL B. A propos des cultures olfactives enfantines et la question du genre: perspectives croisées. In: DELALAND, J.; ARLEO, A. (Éds.). Cultures enfantines/ Children's Cultures. Rennes: Presses Universitaires de Rennes, 2010. p. 313-324.

FERDENZI, C.; WATHELET, O. Odeurs d'adolescences, enjeux des attachements. Cultures et Sociétés, v. 8, p. 99-104, 2008.

FONSECA, Maria Cecília L. O Patrimônio em processo: trajetória da política federal de preservação no Brasil. 2. ed. Rio de Janeiro: UFRJ/MinC-IPHAN, 2005.

GIBBONS, Boyd. The Intimate Sense of Smell. National Geographic Magazine, v. 170, n. 3, p. 324-361, 1986.

GIBSON, James J. The Theory of Affordances. In: GIESEKING, Jen Jack; MANGOLD, William; KATZ, Cindi; LOW, Setha; SAEGERT, Susan (Eds.). The People, Place, and Space Reader. New York: Routledge, 2014. p. 56-60.

GILBERT AVERY, N.; WYSOCKI, Charles J. The Smell Survey: Results. National Geographic Magazine, v. 172, n. 4, p. 514-525, 1987.

HAFSTEIN, Valdimar. Sauvegarde du patrimoine immaterial et gouvernance communautaire. In: 60 ans d'histoire de l'UNESCO. Actes du Colloque International. Paris: Maison de l'UNESCO, 2007. 
HEINICH, Nathalie. La fabrique du patrimoine. "De la cathédrale à la petite cuillère". Paris: Éditions de la MSH, 2009.

HEINICH, Nathalie. Les émotions patrimoniales: de l'affect à l'axiologie. Social Anthropology, v. 20, n. 1, p. 19-33, 2012.

HUYSSEN, Andreas. Seduzidos pela memória. Rio de Janeiro: Aeroplano, 2000.

KAUFMANN, Laurence; CLÉMENT, Fabrice. How Culture Comes to Mind: From Social Affordances to Cultural Analogies. Intellectica, v. 2-3, p. 46-47, p. 221-250, 2007.

MINISTÈRE DE LA CULTURE ET DE LA COMMUNICATION. Une exposition au Palais-Royal. Profession: parfumeur créateur. Culture Communication, n. 198, p. 18-19, fév. 2012.

MUXEL, Anne. Individu et mémoire familiale. Paris: Nathan, 1996.

NIVELEAU, Charles-Édouard. Le concept gibsonien d'affordance: entre filiation, rupture et reconstruction conceptuelle. Intellectica, v. 1, n. 43, p. 159-199, 2006.

NONAKA, Tetsushi; BRIL, Blandine; REIN, Robert. How do stone knappers predict and control the outcome of flaking? Implications for understanding early stone tool technology. Journal of Human Evolution, v. 59, n. 2, p. 155-167, 2010.

SCHAAL, Benoist. Le "matrimoine olfactif": transmissions odorantes entre generations. In: BOILLOT, Francine; GRASSE, Marie-Christine; HOLLEY, André (Éds.). Olfaction \& patrimoine: quelle transmission? Aix-en-Provence: Édisud, 2004. p. 55-77.

SOSA, Ana Maria; FERREIRA, Maria Leticia M. Memoria musealizada: un estudio sobre los procesos de patrimonialización de memorias traumáticas en Uruguay y Brasil. Revista Museologia e Patrimônio, v. 7, n. 1, p. 109-130, 2014.

VAN PRAËT, Michel. Patrimoine culturel et culture scientifique, l'intangible au muse. In: SCHIELE, Bernar (Éd.). Patrimoines et identités. Québec: Musée de la Civilisation et Éditions Multimondes, 2002. p. 65-76.

WATHELET, Olivier. Anthropologie de la transmission des savoirs et savoir-faire sensoriels. Étude de cas: la transmission d'un patrimoine olfactif à l'intérieur de la famille. Thèse (Doctorat) - Université de Nice Sophia Antipolis, 2009.

WATHELET, Olivier. Formes de la transmission familiale des patrimoines olfactifs: partager l'être affecté. In: SCHAAL, B.; FERDENZI, C.; WATHELET, O. (Éds.). Odeurs et émotions. Dijon: Editions universitaires de Dijon, 2012.

WERNER, H. Comparative Psychology of Mental Development. New York: International Universities Press, 1948.

Texto recebido em 11 de outubro de 2015. Texto aprovado em 11 de outubro de 2015. 